Article

\title{
Optimal Battery Sizing for Electric Truck Delivery
}

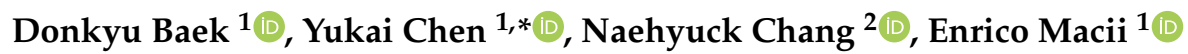 \\ and Massimo Poncino ${ }^{1}$ (D) \\ 1 Politecnico di Torino, 10129 Torino, Italy; donkyu.baek@polito.it (D.B.); enrico.macii@polito.it (E.M.); \\ massimo.poncino@polito.it (M.P.) \\ 2 Korea Advanced Institute of Science and Technology, Daejeon 34141, Korea; naehyuck@cad4x.kaist.ac.kr \\ * Correspondence: yukai.chen@polito.it
}

Received: 27 December 2019; Accepted: 30 January 2020; Published: 6 February 2020

\begin{abstract}
Finding the cost-optimal battery size in the context of parcel delivery with Electric Vehicles (EVs) requires solving a tradeoff between using the largest possible battery (so as to maximize the number of deliveries over a given time) and the relative costs (initial investment plus the unnecessary increase of the truck weight during delivery). In this paper, we propose a framework for the optimal battery sizing for parcel delivery with an electric truck; we implement an electric truck simulator including a nonlinear battery model to evaluate revenue, battery cost, charging cost, and overall profit for annual delivery. Our framework finds the cost-optimal battery size for different parcel weight distributions and customer location distributions. We analyze the effect of battery sizing on the profit, which is up to $56 \%$.
\end{abstract}

Keywords: electric truck delivery; cost-optimal battery sizing; framework; EV powertrain model; dynamic battery model; battery aging model; battery depreciation cost

\section{Introduction}

Electric Vehicles (EVs) are expected to progressively replace conventional Internal Combustion Engine Vehicles (ICEVs) because of their high energy efficiency and zero Green House Gas (GHG) emission. Recently, the landscape of EVs has extended to domains such as electric racing cars, buses, and trucks, generating new application domains and new problems. The case of electric trucks is particularly interesting as trucks account for about $60 \%$ of the overall freight transportation [1]. Tesla announced that electric trucks will replace ICE trucks in the near future because of several merits of electric propulsion with respect to traditional propulsion [2].

This work focuses on the problem of parcel delivery on a small/medium geographical scale (city or district, less than a $100 \mathrm{~km} \times 100 \mathrm{~km}$ area). In this context, interesting economic tradeoffs related to the battery sizing arise. While a larger battery size guarantees a longer driving range and thus the delivery of as many packages as possible during working hours, on the other hand, a too large battery increases the total vehicle weight, which causes higher energy consumption. On the other hand, if the battery size is reduced by decreasing the number of battery cells in parallel, the total current of the battery pack decreases because of the reduced weight. However, the amount of current drawn from each battery cell becomes larger. The battery State Of Charge (SOC) decreases quickly, and discharging efficiency is reduced for a battery with low SOC [3]. Furthermore, a relatively high discharging current on each battery cell accelerates battery aging by increasing the number of charging and discharging cycles, resulting in replacing the battery earlier. Therefore, it is important to identify the optimal battery size for a given delivery task so as to maximize overall profit including battery and management cost because battery efficiency, aging, and purchasing cost strongly depend on the battery size. 
The design optimization of the electric vehicle has been studied for a long time [4-7]. Vehicle simulators have been used to estimate the energy consumption by a given combination of vehicle components such as battery and motor size. From the energy consumption and related design setup, design frameworks estimate the overall initial vehicle investment cost, management cost by battery charging, and battery replacement cost by battery aging issue. However, most previous work focused on the vehicle design on a few given driving profiles and rarely considered the relationship between the overall profit and delivery missions. For example, we can deliver more packages with a lager battery, which increases the revenue by paying more electricity cost. Furthermore, the revenue and cost vary depending on the weight of the packages and the area of delivery coverage. Modern automobile manufacturing systems allow multiple different trims such as battery size on the same assembly line. For example, Tesla suggests several trims (standard range, long range, and performance) for the same Model 3. Therefore, users can choose the optimal trim for their purpose.

In this paper, we propose a framework to determine the optimal battery sizing for an EV truck for a given set of delivery tasks. The framework relies on accurate models of (i) the power flow, specifically of the EV powertrain, of battery discharge, and battery aging and (ii) the total revenues, which includes a battery cost and a charge electricity cost model, both depending on the resulting power flow. One important variable in our evaluation is the number of working hours, which sets an upper bound to the number of deliveries in a day and limits even further the need for excessively large batteries.

We report results for various delivery request profiles having various distributions of package weight and delivery distance, as well as different numbers of daily working hours. We find the optimal battery size improving the profit up to $56 \%$ for the same delivery request, and we also identify configurations for which profit becomes negative.

The paper is organized as follows. Section 2 describes the motivation for framework for the optimal battery sizing for parcel delivery with an electric truck. Section 3 introduces the background and presents related work. Section 4 describes the system model of our proposed framework, which includes the general scenario of our method, electric truck power models, and cost models for delivery. Section 5 presents the details of the delivery problem. Sections 6 describes the implementation and simulation results of the optimal battery sizing methods by delivery requests, working hours, the time horizon, and in the case study. Finally, Section 7 concludes the paper.

\section{Motivation}

Consider a scenario in which we have a (possibly infinite) number of deliveries to be served in a pre-defined order over a given time horizon $T$ (e.g., a day, month, or year). We want to analyze the economic balance of the delivery considering that we have at most a number $T_{w h}$ of hours per day available for delivery; at the end of the working hours, the battery is fully re-charged to $100 \%$ before the start of the next day. The costs involved are (1) the battery purchase cost plus its depreciation and (2) electricity cost for battery re-charge (we did not consider the vehicle purchase cost, as we assumed the fleet was already present). Revenues come from shipping fees.

Suppose now that we want to explore how costs and revenues change for different battery sizes $B$. A pictorial view of the analysis is reported in Figure 1, referring to some value of $T$.

Initially, for small values of $B$, only a small number of deliveries can be completed over a day (Region I); in this region, increasing the battery size will progressively allow completing more deliveries (linear increase of revenues), at the price of a linear increase in the battery cost, essentially due to purchase cost.

The battery depreciation factor increases moderately in this region; as a matter of fact, as the battery capacity is limited, the truck will deliver as many packages as possible and arrive at the depot at the end of the service. Therefore, each day approximately corresponds to one entire cycle (The actual charging cost will exhibit a more irregular profile. Figure 1 just reports the qualitative trend). 


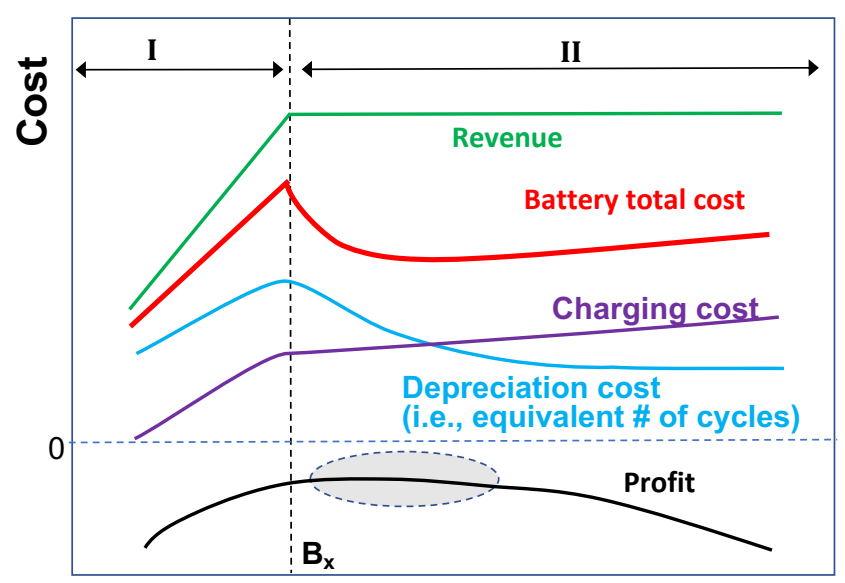

Battery size (B)

Figure 1. Motivational example.

When reaching some battery size $B_{x}$, we will deliver the maximum amount of packages allowed by the working hours constraint $T_{w h}$. After this point, increasing the battery size will not increase the revenue, which will saturate. Apparently, as a result of a larger battery, the cost should increase and thus the profit (revenue minus total cost) decrease accordingly. However, as the number of delivered packages is constant (the analysis refers to a given internal $T$ ), the pre-defined delivery schedule will deplete this larger battery less, and the vehicle will arrive at the depot with more residual charge. Therefore, one working day will correspond to an increasingly smaller fraction of a charge cycle. As a result, the total battery cost will decrease because of (i) a smaller depreciation (smaller number of equivalent charge cycles) and (ii) a smaller re-charge cost (the truck arrives with more residual charge).

There is however another variable to consider in this analysis: a larger battery will increase the total vehicle weight (vehicle + payload) and thus its power consumption, thus depleting the battery more. Thus, the benefits from having a larger battery described above are progressively lost as we increase the battery size: after some value, the increase in vehicle power consumption will start decreasing the residual charge at the end of the service, and total cost will start increasing again.

The bottom line is that, as revenue stays constant and total cost has a minimum, there will be an optimal battery size that maximizes the profit; this is the area highlighted in the oval on the profit curve and the objective of our work.

\section{Background and Related Work}

There have been several research works deriving optimal vehicle designs using model-based vehicle simulators. The work in [4] proposed a design optimization methodology for a fuel cell powered hybrid and plug-in hybrid electric bus. There were various size candidates for electric motors, fuel cells, and battery packs in a library, which included specifications of each component. The authors performed vehicle driving simulations by changing the electric motor, fuel cell, and battery pack to estimate $\mathrm{CO}_{2}$ emission, fuel, and investment cost. Using the genetic algorithm, they looked for the best component combination minimizing the emission and cost.

The work in $[5,6]$ considered a battery aging issue in the battery design methodology. The authors proposed design methodologies for optimal sizing of the Hybrid Energy Storage System (HESS) used in an electric vehicle to minimize both of the total HESS cost and battery degradation for a given driving profile. As the size of supercapacitor increases, the battery aging is reduced by decreasing the battery cycle time. However, the total storage cost increases. On the other hand, a small size of the supercapacitor increases the amount of battery usage during the vehicle driving, so the battery degradation increases, which accelerates the battery degradation. The authors derived the Pareto front in terms of total HESS cost and the battery state of health. 
The work in $[7,8]$ analyzed the cost-of-ownership of a medium-duty hybrid electric truck business considering initial investment cost, fuel economy, and battery replacement cost by battery degradation. The proposed framework in [8] explored a design space on various engine and electric motor power, the size of the battery, payload, etc., under some given driving cycles. The framework estimated the above overall costs and payback period by a given revenue in each component combination. The authors in [7] minimized the total cost-of-ownership of fuel cell powered trucks by changing the size of hydrogen storage and battery capacity. The optimization was done under minimum acceleration, top speed, and driving range constraints.

Most previous work considered only a given driving cycle to estimate the battery cycle time and cost of ownership. In the truck delivery problem, the driving cycle is changed by the number of deliverable packages and related delivery route, which depend on the size of the battery. A truck with a larger battery pack can drive a longer distance with heavier payloads and increases delivery revenue. Furthermore, previous work did not consider the nonlinearity of lithium-ion battery. The battery efficiency is highly affected by the amount of a load current and determined by the number of parallel connections of the battery pack. In addition, the battery degradation reduces the battery capacity and total amount of deliverable packages.

Compared with the previous work, we propose a new framework for the optimal battery sizing by delivery tasks. We implement a daily driving cycle based on delivery tasks and battery size. Based on the driving cycle, the framework calculates annual revenue, battery cost, and overall profit. To estimate the battery cost and electricity cost accurately, we use models of nonlinear lithium-ion battery discharging and aging characteristics.

\section{System Modeling}

\subsection{System Model}

We considered a scenario where we had a fleet of EV trucks and a set of delivery tasks, and we wanted to choose an optimally-sized battery for a given truck based on its list of delivery tasks (Figure 2).

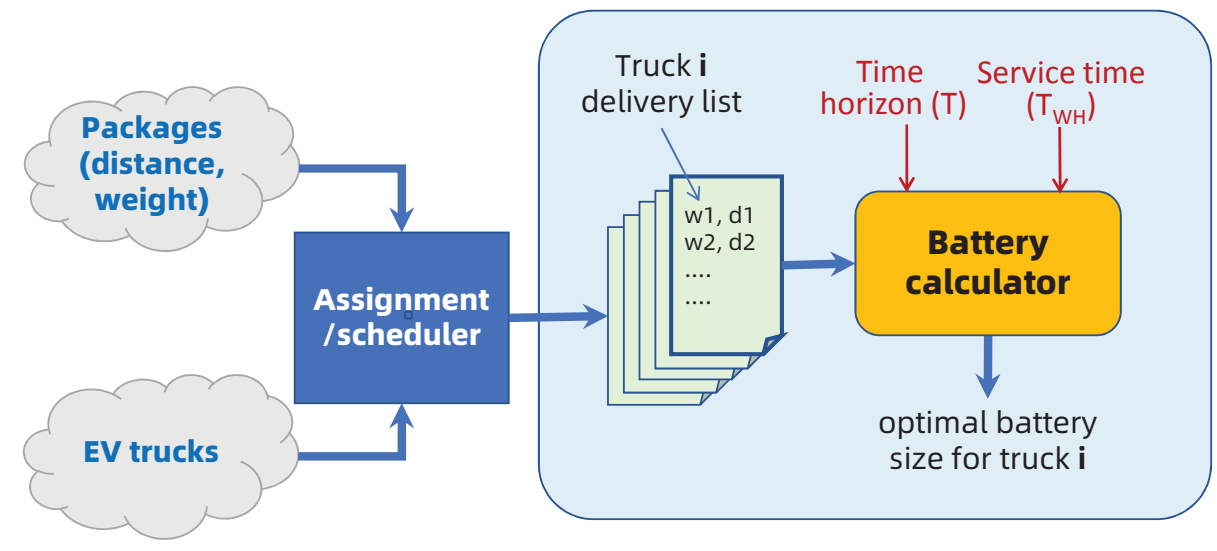

Figure 2. General scenario of our method.

We did not consider here the issue of the scheduling and/or the assignment of packages to trucks, but rather assumed that our tool operated downstream of such an assignment algorithm. This was not a limitation, as it allowed running any existing algorithm to determine a good schedule according to different metrics [9].

Our cost-optimal battery size calculator (shown in the rounded rectangle in figure) took therefore a list of delivery tasks (weight and distance from a depot) and considered each truck independently of the others. Notice that the tool took two parameters: the overall time horizon of the analysis $T$ and the 
number of daily working hours $T_{W H}$, which were essential to perform the economical assessment that determined the battery size. It was therefore assumed that, for each truck, a list of deliveries long enough to cover $T$ was available from the scheduler.

Notice however that this latter requirement did not imply knowing in advance the whole delivery schedule for the entire horizon $T$. The task list could be generated dynamically and added to the task list as a log file. As a matter of fact, our method did not aim at altering the delivery route, but it just determined the optimal battery pack to use for a given truck. Therefore, it was sufficient that the delivery list for a single day be provided to our sizing algorithm at the beginning of the service, which a pretty reasonable requirement for a typical logistic scenario.

\subsection{Power Consumption Models}

\subsubsection{Electric Truck Powertrain Model}

When a vehicle drives on a road, four resistances act on the vehicle: rolling resistance $F_{R}$, gradient resistance $F_{G}$, inertia resistance $F_{I}$, and aerodynamic resistance $F_{A}$. Power consumption to overcome the resistances $P_{\text {res }}$ is a function of torque $T$ and angular speed $\omega$. All resistances except $F_{A}$ are linearly proportional to vehicle mass $m$.

$$
\begin{gathered}
P_{r e s}=T \omega=F d s / d t=\left(F_{R}+F_{G}+F_{I}+F_{A}\right) v \\
F_{R} \propto C_{r r} m g, F_{G} \propto m g \sin \theta, F_{I} \propto m a, F_{A} \propto \frac{1}{2} \rho C_{d} A v^{2} .
\end{gathered}
$$

Practical power consumption by electric motors $P_{E V}$ is the sum of the power to overcome the resistances $P_{\text {res }}$ and power loss $P_{\text {loss }}$ from the motor rotation [10]. The $P_{E V}$ depends on the total weight of EV $m$, road slope $\theta$, vehicle speed $v$, and acceleration $a$ :

$$
P_{E V}=P_{\text {res }}+P_{\text {loss }}=f(m, \theta, v, a) .
$$

\subsubsection{Non-Linear Dynamic Battery Model}

The model of the battery pack must be able to account for the load current and SOC variations of the usable battery capacity accurately. A single cell was modeled with a circuit-equivalent model that considered the capacity dependency on the current magnitude and dynamics [3]. The circuit-equivalent model as shown in Figure 3 consisted of a battery lifetime model on the left-hand side and a battery voltage model on the right-hand side, respectively. In the battery lifetime model, a capacitor $C$ represented the battery capacity, and a current generator $I_{b a t t}$ represented load current. Two voltage generators $V_{\text {lost }}\left(f_{\text {load }}\right)$ and $V_{\text {lost }}\left(I_{\text {load }}\right)$ were used to consider dependencies on the amount and frequency of the load current. Both a larger amount and higher frequency of the load current decreased the state of charge (SOC). Battery voltage $V_{b a t t}$ on the right-hand side was then calculated based on the SOC and battery internal resistance and capacitance.

On the right-hand side, a voltage-controlled voltage generator expressed the non-linear dependence of battery open-circuit voltage $V_{o c}$ (SOC). The RC network modeled the battery impedance, by exposing a series resistance $R(\mathrm{SOC})$ that modeled the internal resistance and two RC blocks tracking the short-term $\left(R_{S}, C_{S}\right)$ and long-term $\left(R_{L}, C_{L}\right)$ time constants of a step response.

From this battery cell model, we built a battery pack model by simply scaling all parameters according to the series/parallel connection. Even though there were some mismatches on the connection, this battery pack model was still more accurate than a linear model that neglects state-dependent battery characteristic.

Given this model, we tracked the battery SOC and battery voltage $V_{\text {batt }}$ by applying the drawn power (as current and voltage waveforms) from the electrical motors. In the most general case, there was a non-ideal power conversion between the electrical motor and the battery. In this case, we assumed a constant conversion efficiency, i.e., $P_{\text {batt }}=P_{\text {motor }} \cdot \eta$. 


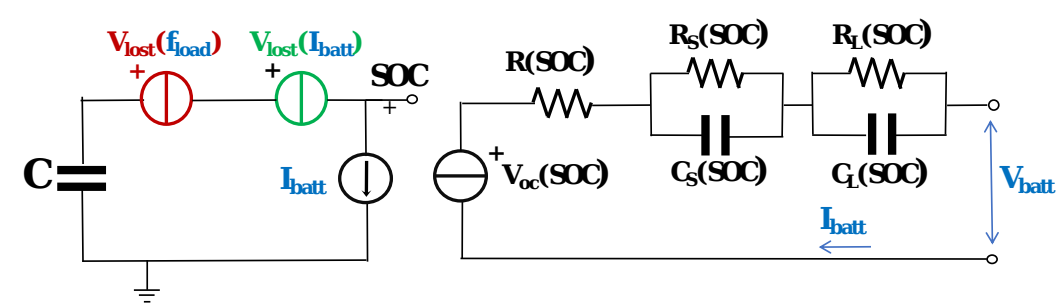

Figure 3. Adopted circuit-equivalent model for the battery cell.

\subsection{Cost Models}

\subsubsection{Battery Depreciation Model}

Effective number of cycles $N_{c y c}$ is the amount of charging and discharging energy divided by a nominal battery capacity $Q_{n o m}$. As $N_{c y c}$ increases, battery capacity decreases because of the battery aging effect. If the battery capacity becomes less than the minimum battery capacity, the battery should be replaced with a new one by paying battery cost. Battery depreciation $\operatorname{cost} C_{\text {depr }}$ is used to reallocate the overall battery cost over the life span of the battery, which depends on the charging and discharging of the battery. Therefore, $C_{d e p r}$ is a function of $N_{c y c}$, which is $N_{c y c}$ divided by a product of the maximum charging and discharging cycles of the battery $N_{c y c, m a x}$ and an average battery replacement $\operatorname{cost} C_{b a t t}$. The equations of $N_{c y c}$ and $C_{d e p r}$ are:

$$
N_{c y c}=\frac{\int_{0}^{T}|I(t)| d t}{2 Q_{n o m}} \text { and } C_{d e p r}=\frac{N_{c y c}}{N_{c y c, m a x} C_{b a t t}} .
$$

\subsubsection{Electricity Charging Model}

There are several battery charging models and optimal battery charging and management methods considering battery degradation [11,12]. However, in this paper, we mainly focused on electricity charging cost and used a simple battery charging model to calculate charging cost easily.

The electricity charging cost $C_{\text {elec }}$ for $n$ deliveries is:

$$
C_{\text {elec }}=\sum_{i=1}^{n} D O D_{i} Q_{n o m} V_{\text {batt }} C_{\text {unit }}
$$

where $D O D_{i}, V_{\text {batt }}$, and $C_{\text {unit }}$ are a battery's depth of discharge for the $i^{\text {th }}$ delivery, a battery pack's voltage, and the average unit cost for electricity, respectively.

\subsubsection{Delivery Revenue Model}

In the most general case, the delivery revenue is a non-decreasing function of parcel weight (more typical), delivery distance, or a combination of both; i.e., for the $i^{\text {th }}$ delivery, $C_{r e v, i}=f\left(w_{i}, d_{i}\right)$. Our framework did not make any assumption on the type of function.

\section{Delivery Problem}

Figure 4 describes the delivery and battery sizing algorithm. It took as inputs (1) the list $\mathcal{T}=$ $\left[\tau_{1}, \ldots, \tau_{n}\right]$ of the $n$ delivery tasks, where a task $\tau_{j}$ is characterized by a package weight $w_{j}$ and a location in terms of its coordinates $x_{j}$ and $y_{j}$ in a plane; (2) the overall time horizon $T$ and the working hours bound $T_{w h}$. 


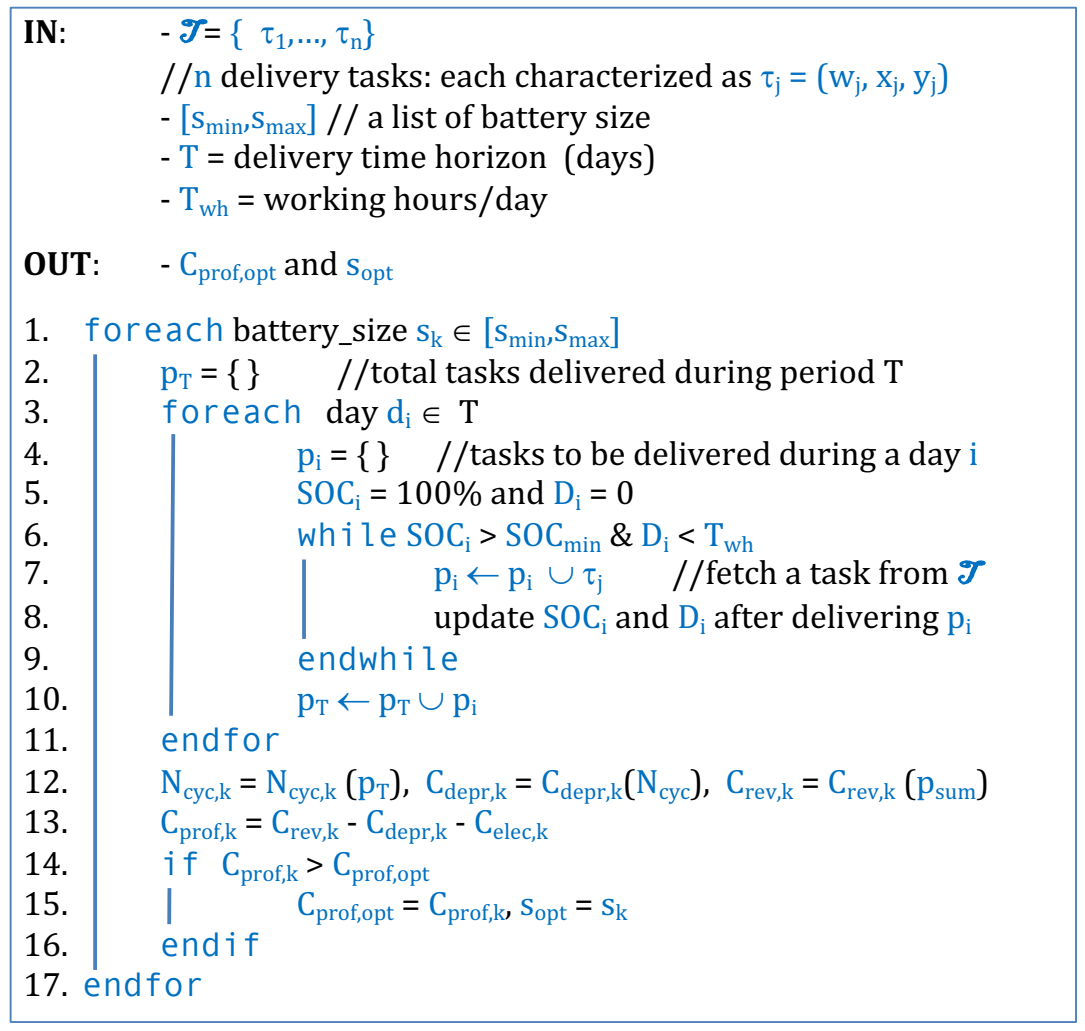

Figure 4. Delivery tasks.

The objective of this algorithm is to estimate the profits of the delivery business $C_{\text {prof }}$ by battery size; this is achieved by simulating the whole delivery schedule $\mathcal{T}$ for different battery sizes within a given range $\left[s_{\min }, s_{\max }\right]$. For each battery size $s_{k}$ (Line 1$)$, the total set of delivered tasks over $T p_{T}$ is calculated by accruing the deliveries completed $p_{i}$ in each day $d_{i}$. The truck departs from the depot and returns to the depot after delivering as many packages as possible $p_{i}$ as allowed by the given battery capacity and working hour of a day (Lines 6 to 9). A delivery task $\tau_{i}$ can be accomplished as long as the battery SOC $S O C_{i}>S O C_{\min }$ and the total daily driving time $D_{i}<T_{w h}$. As soon as one of these two conditions does not hold, the daily delivery is considered as over, and we start processing the next day. Notice that Line 5 is reset to $100 \%$ (i.e., the battery is re-charged by the start time of the next day) and $D_{i}$ to 0 .

After determining delivery schemes for the number of days covered by $T$, we calculate $N_{c y c, k}$, $C_{d e p r, k}$, and $C_{r e v, k}$ for the current battery size $s_{k}$ (Line 12). $C_{p r o f, k}$ is obtained from $C_{r e v, k}$ subtracted by the sum of $C_{\text {depr, }, k}$ and $C_{\text {elec, }, k}$ (Line 13). If $C_{\text {prof }, k}$ is higher than current maximum profit $C_{\text {prof,opt, }}$ we update the current optimal battery size $s_{\text {opt }}$. The process is then restarted for another battery size.

\section{Simulation Results}

\subsection{Simulation Setup}

\subsubsection{Powertrain Model}

We adopted a powertrain model of a Tesla Semi truck from [9]. The coefficients of Equation (2) were obtained from an implementation of an ADVISOR (Advanced vehicle Simulator) vehicle model and variable regression by a number of simulations as described in [10]. The powertrain consisted of four Model 3 electric motors; each motor was a three-phase AC permanent magnet electric motor, with a maximum power of $192 \mathrm{~kW}$ from 4700 to $9000 \mathrm{RPM}$, and the maximum torque was $410 \mathrm{Nm}$ below 4500 RPM, respectively [13,14]. 


\subsubsection{Battery Model}

We assumed that four electric motors were powered by four battery packs. Each battery pack was composed of four modules that were connected in series; each module consisted of Panasonic NCR18650B $3400 \mathrm{mAh}$ lithium battery cells arranged in a 24 series configuration [15]. The number of parallel connection in a module was variable in the following simulations. Table 1 specifies nominal and cut-off voltages of each cell, each module, and the whole battery pack.

Table 1. Electrical parameters of the battery pack.

\begin{tabular}{crrr}
\hline Parameters & Cell & Module & Whole Pack \\
\hline Nominal Voltage & $3.6 \mathrm{~V}$ & $86.4 \mathrm{~V}$ & $345.6 \mathrm{~V}$ \\
Cut-off voltage & $2.75 \mathrm{~V}$ & $66.0 \mathrm{~V}$ & $264.0 \mathrm{~V}$ \\
\hline
\end{tabular}

We built our single cell battery discharging model based on the measurement data by adopting the method described in [3]. We assumed battery cells in the pack to be ideally balanced in the following experiments, then built the battery pack model as described in Section 4.2.2.

\subsubsection{Battery Aging and Replacement}

There are various battery aging models considering the prediction of calendar and cyclic modes. In this paper, we chose a simple battery cyclic aging model using a lookup table as a function of charging and discharging cycles. This lookup table-based aging model was good enough to estimate the decrease in battery capacity and replacement cycle. The battery health degraded by the number of full charging/discharging cycles, which was specified in the datasheet of Panasonic NCR18650B: the cell capacity decreased from $3400 \mathrm{mAh}$ to $2250 \mathrm{mAh}$ after 500 full charge-discharge cycles, after which the replacement of the battery pack was recommended. Replacement of one battery module in Model 3 cost from 5 k-7 k USD according to a comment by Elon Musk [16]. Based on this datum, we assumed that the average cost for the replacement of one battery cell $C_{\text {batt }}$ as $\approx 5.5$ USD.

\subsubsection{Charging Costs}

Since the available time for re-charge was longer than 12 hours, we assumed a Level 2 charging in the depot. We used a constant electricity rate of $0.1 \mathrm{USD} / \mathrm{kWh}$, which was the average charging cost in the U.S.; a fixed cost was reasonable as the charging should occur systematically at night time. In any case, our framework could be easily extended to consider other electricity cost models (e.g., time of day).

\subsubsection{Delivery Revenue}

We adopted a shipping rate from Deutsche Post DHL [17] specified in Table 2. In the case of delivery distance, we assumed that all requests were "local" deliveries; thus, revenue did not depend on distance. Again, we could easily incorporate a distance-dependent delivery cost should distances increase.

Table 2. Shipping rate of Deutsche Post DHL [17].

\begin{tabular}{cccc}
\hline Package Weight & Revenue & Package Weight & Revenue \\
\hline Less than $0.5 \mathrm{~kg}$ & EUR 11.9 & Less than $5.0 \mathrm{~kg}$ & EUR 20.9 \\
Less than $1.0 \mathrm{~kg}$ & EUR 12.9 & Less than $10 \mathrm{~kg}$ & EUR 25.9 \\
Less than $2.0 \mathrm{~kg}$ & EUR 14.9 & Less than $20 \mathrm{~kg}$ & EUR 30.9 \\
Over $20 \mathrm{~kg}$ & EUR 45.9 & & \\
\hline
\end{tabular}




\subsection{Impact of Delivery Requests on Profit}

In this first simulation, we considered $T=1$ year and $T_{w h}=12$ hours and evaluated the annual profits with respect to battery size for a set of different delivery tasks. The deliveries referred to:

- a set of customer locations in four different areas of $20 \times 20 \mathrm{~km}, 30 \times 30 \mathrm{~km}, 50 \times 50 \mathrm{~km}$, and $70 \times 70 \mathrm{~km}$, with $(\mathrm{x}, \mathrm{y})$ coordinates uniformly distributed within the relative ranges;

- a set of package weights, according to two distributions in the [0,30 kg] range: uniform and half-normal, in order to account for the case in which the majority of deliveries consisted of light packages and heavy ones were the exception. Figure 5a,b shows the two weight distributions used.

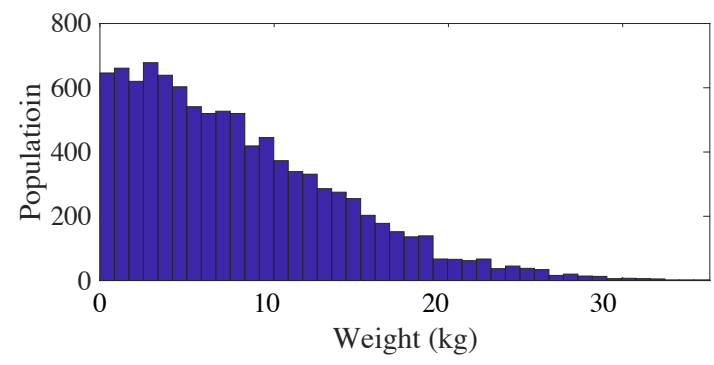

(a)

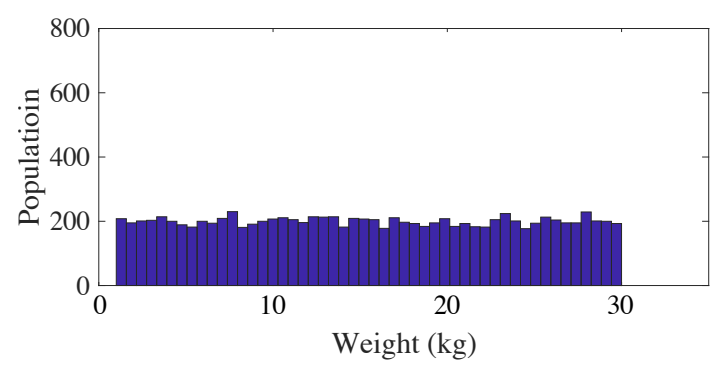

(b)

Figure 5. Package weight distributions.

For the estimation of the truck power consumption, we assumed that the movements between destinations all occurred at a constant speed representing an average speed in the area of delivery; specifically we used $20.76 \mathrm{~km} / \mathrm{h}$, which was the average rush-hour speed on urban arterial streets in San Francisco [18]. Despite being an approximation (speed variations had some impact on the battery energy consumption), for the purpose of our analysis, the assumption was reasonable. Moreover, adding time-dependent speed values would yield results too dependent on the specific speed profile. Our framework could anyway be adapted to actual speed traces or estimates using traffic-aware navigation data (e.g., as done in [10]).

Finally, to take into account also the dependency on input data, we generated 10 instances of the delivery task set $\mathcal{T}$.

Figure 6 shows the simulation results. The figure shows eight plots: the four in the left column refer to the half-normal package weight distribution and the ones in the right column to the uniform weight distribution. Rows, from top to bottom, refer to different delivery areas: $20 \times 20 \mathrm{~km}, 30 \times 30 \mathrm{~km}$, $50 \times 50 \mathrm{~km}$, and $70 \times 70 \mathrm{~km}$, respectively. Each plot contains 10 curves, one for each different task set. The scale of the battery size on the $\mathrm{X}$-axis is given as the number of battery cells. Each tick corresponds to 40 cells in parallel. Labels are however given in kAh for a numeric equivalent in terms of capacity.

The first observation was that all curves exhibited the shape of the conceptual one in Figure 1;

As the delivery area became larger (from Figure 6a-d), annual profit obviously decreased, since the number of customers that could be visited during a day was reduced. As the gap between revenue and battery depreciation cost became smaller because of decreasing annual revenue (as in the case of larger delivery areas), the effect of an appropriate battery sizing on the annual profit became more important. For instance, in Figure $6 \mathrm{~h}$, the choice of an optimal battery size yielded a profit that could be from $37 \%$ to $95 \%$ (depending on the specific instance) larger than the one achieved using the maximum battery size (which would be the intuitive choice, as a result of larger delivery areas).

Concerning the impact of weight distribution, it was clear that, for a given delivery area, profits were higher when the average weight was larger, as in the uniform case. For the semi-Gaussian case, profits became losses regardless of the battery size and of the task set instance.

The most interesting result was that, independent of the weight distribution or delivery area, the maximum profit was for all cases approximately in the same region around $1500 \mathrm{Ah}$ (marked as 
a red rectangle). Although for smaller delivery areas (Figure $6 \mathrm{a}, \mathrm{b}, \mathrm{e}, \mathrm{f}$ ), one could be tempted to opt for the smallest possible size just after the "saturation" of the profit (e.g., around $800 \mathrm{Ah}$ for these four scenarios), this was not the true optimum: our analysis already included the investment and obsolescence cost of the battery.

The average profit gain with respect to using the maximum battery size ranged from $3 \%$ up to $56 \%$ depending on the scenario. The minimum gain was obtained from Figure $6 a$ and the maximum gain from Figure $6 \mathrm{~h}$.

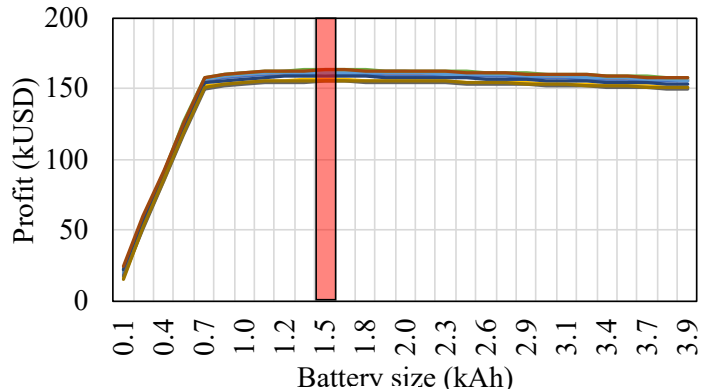

(a)

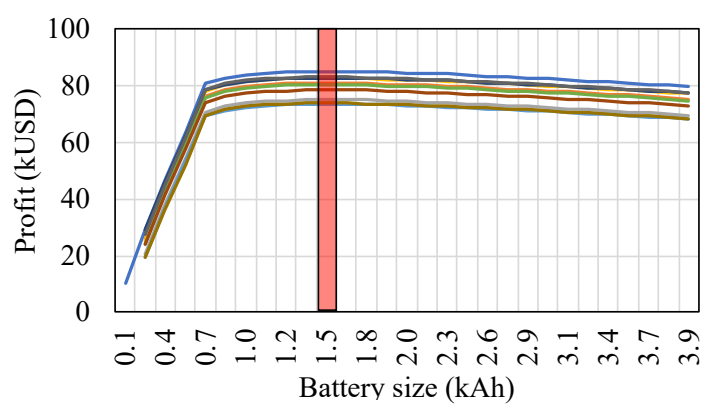

(b)

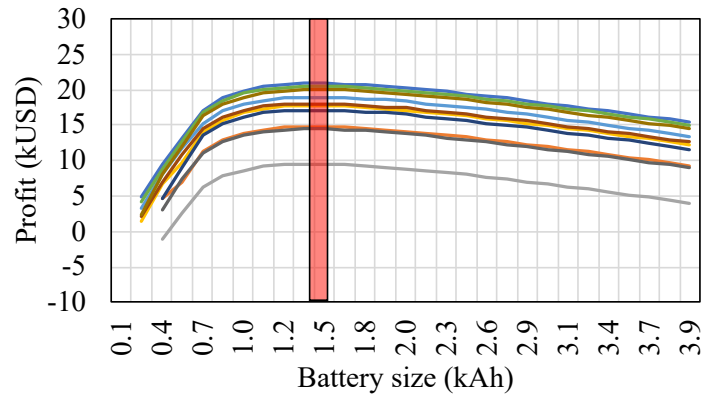

(c)

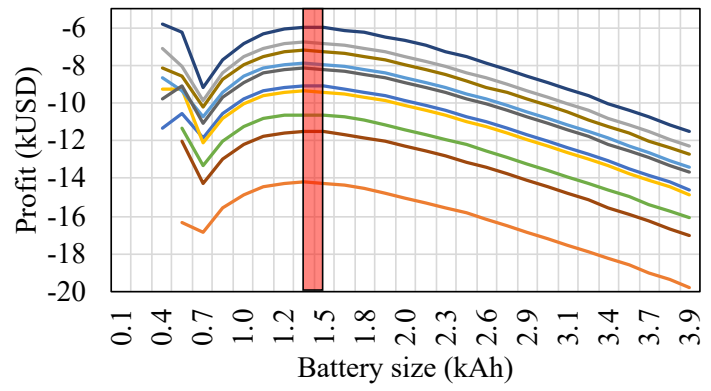

(d)

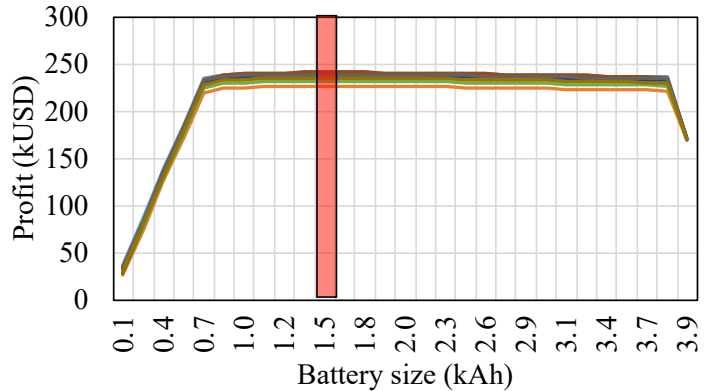

(e)

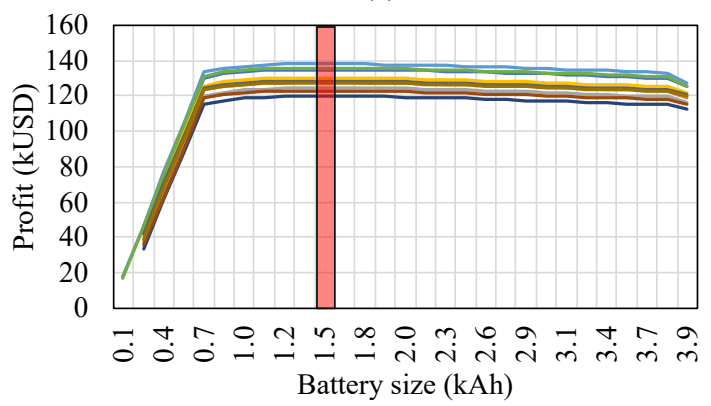

(f)

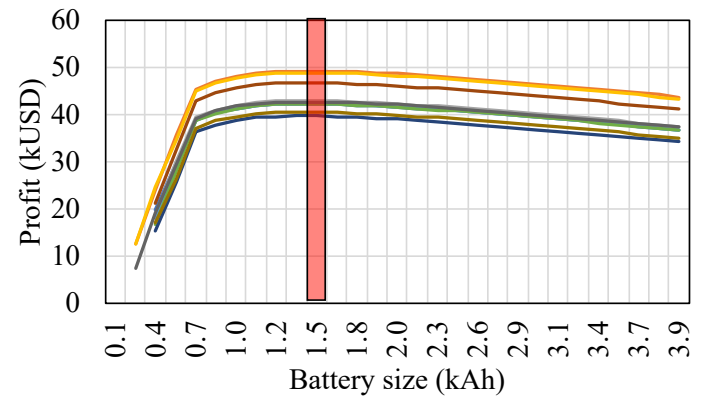

(g)

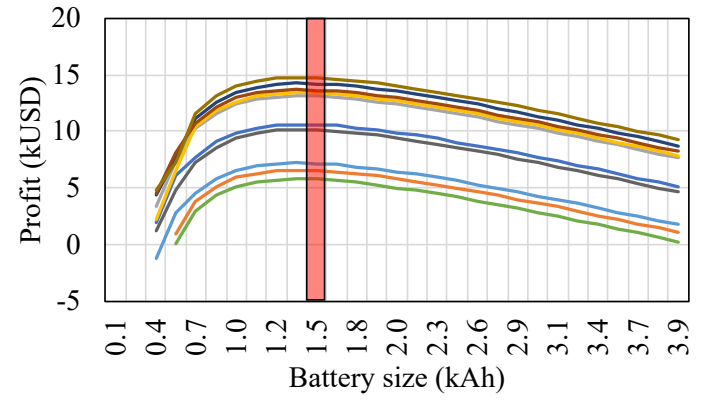

(h)

Figure 6. Annual profits by distributions of package weight and customer location.

In general, profit by an optimally-sized battery was observed when the battery size was enough for a delivery during working hours because, as shown in Figure 1, revenue saturated after the knee 
point of the curve, and in this region, it was the battery depreciation cost that affected the overall profit. The battery depreciation cost was smallest when the total driving distance was shortest. Because the truck kept driving during working hour (neglecting the short unloading time), the total driving distance was determined by working hour. Therefore, the optimal battery size was approximately the same for a given value of $T_{W H}$.

\subsection{Annual Profit by Working Hours}

A key parameter in our analysis was the value $T_{W H}$. We clearly expected that profits would increase as we extended this value as more requests could be accomplished over a given interval $T$. On the other hand, as trucks had to re-charged and battery capacity was limited, we could not increase $T_{W H}$ arbitrarily since there was a limit to the time between re-charge.

Figure 7 plots the annual profits by battery size from six working hours to 12 working hours. For simplicity, we refer to a specific instance of the cases described in the previous section, namely a $50 \times 50 \mathrm{~km}$ delivery area using the half-normal distribution of package weights.

We can see that the optimal battery size differed with $T_{W H}$ and expectedly decreased with shortened working hour windows. The optimal battery sizes maximizing the profit were $1.0 \mathrm{kAh}$, $1.1 \mathrm{kAh}, 1.2 \mathrm{kAh}$, and $1.4 \mathrm{kAh}$ when the working hours were $6,8,10$, and 12, respectively. For example, $1.0 \mathrm{kAh}$ was the optimal battery size for $T_{W H}=6$, significantly smaller than the $1.4 \mathrm{kAh}$ of the $12 \mathrm{~h}$ case.

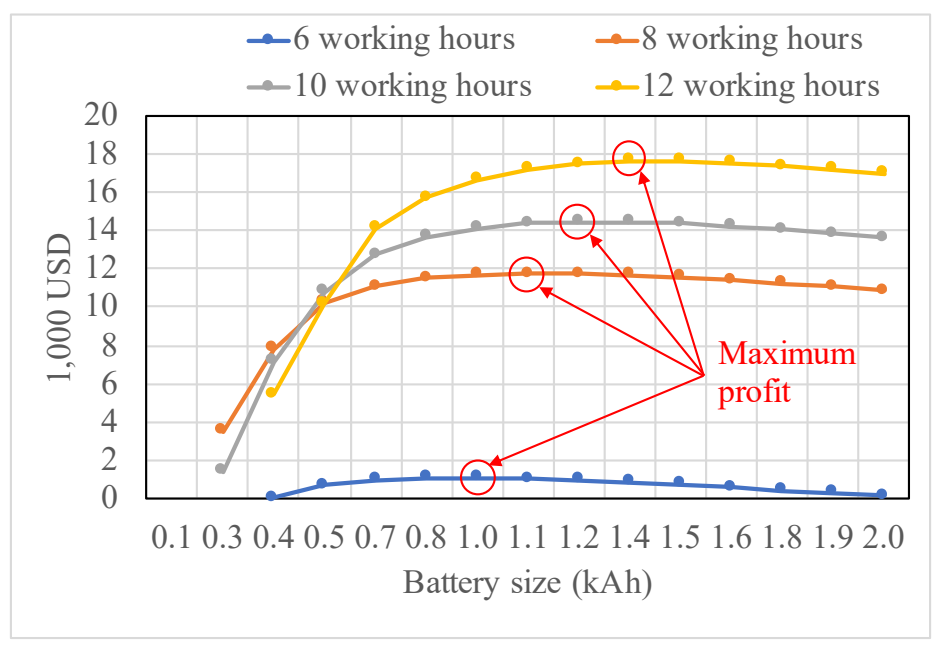

Figure 7. Annual profit by working hour.

\subsection{Dependency on the Time Horizon}

Another interesting parameter to be considered is the time horizon $T$. Intuitively, benefits will increase for larger values of $T$; however, we should also take into account that smaller battery sizes and increased time horizon might incur a need for one or more replacements of the battery pack, which would clearly impact the cost analysis. This issue was not considered in the analysis carried out in Section 2.

We generated thus a number of delivery problems with the value of $T$ of one week, one month, one year, and several years; as before, for each $T$, we generated 10 random instances and estimated the profit by battery size. In this simulation, we considered that customer locations were uniformly distributed in a $50 \mathrm{~km}$ by $50 \mathrm{~km}$ area with a set of half-normal package weight distribution.

Figure 8 shows the profit as a function of battery size, for the different $T^{\prime}$ s. When $T \leq 1$ year (from Figure $8 \mathrm{a}-\mathrm{d}$, the trend was similar, just the total accrued benefit scaled proportionally; the optimal battery size resulted in about 200-400 \$ profit for $T=1$ week, 500-2200 \$ for 1 month, 5-9 k\$ for 6 months, and $13-20 \mathrm{k} \$$ for 1 year.

As we increased $T$ to values that might result in wearing out of the battery (typically after 2-3 years, corresponding to approximately 1000 charge cycles), the shape of the profit curve changed. 
Depending on the battery size, one or more replacements might be needed. When $T=3$ years, as shown in Figure 8e, the battery needed to be replaced if its size was smaller than $1.2 \mathrm{kAh}$. If we increased $T$ to five years (Figure 8f), two replacements were needed for sizes smaller than $1.1 \mathrm{kAh}$ and one replacement for size smaller than $2.3 \mathrm{kAh}$. These events resulted in dips in the profit curves, which were more evident when a larger battery was replaced, and that caused the presence of local minima and maxima.

This analysis made our framework even more useful, as it made the result less trivial. For instance, in Figure $8 \mathrm{f}$, the two local maxima (around $B \approx 1 \mathrm{kAh}$ and $B \approx 1.5 \mathrm{Ah}$ ) have similar profit values $(\approx 80 \mathrm{k} \$)$ in spite of requiring two replacements for the first case and only one in the second case. In this case, other considerations might apply (e.g., using a smaller battery might be preferred for some reason).

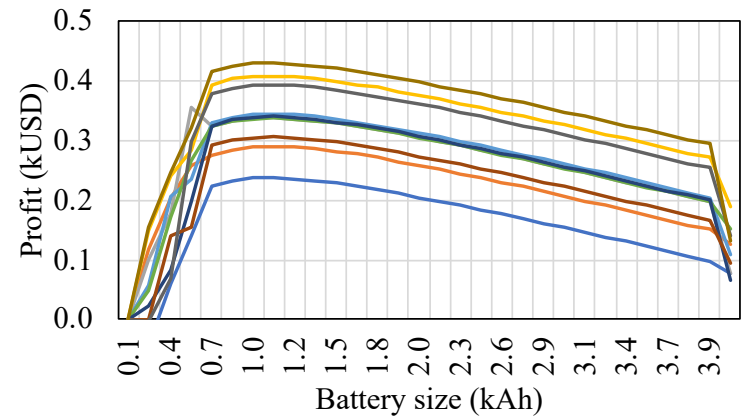

(a) $T=1$ week.

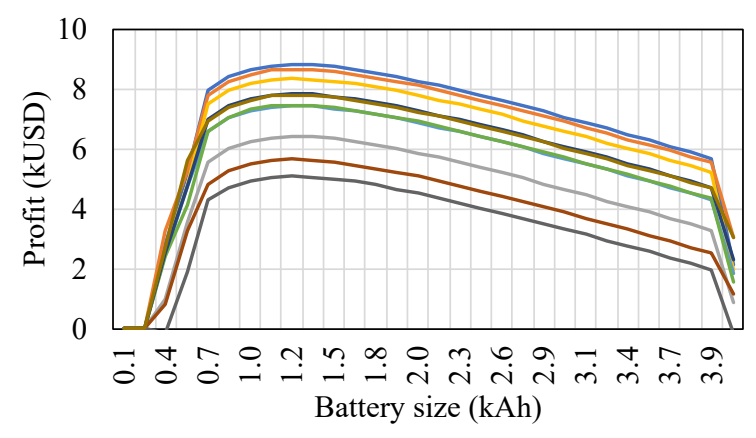

(c) $T=6$ months.

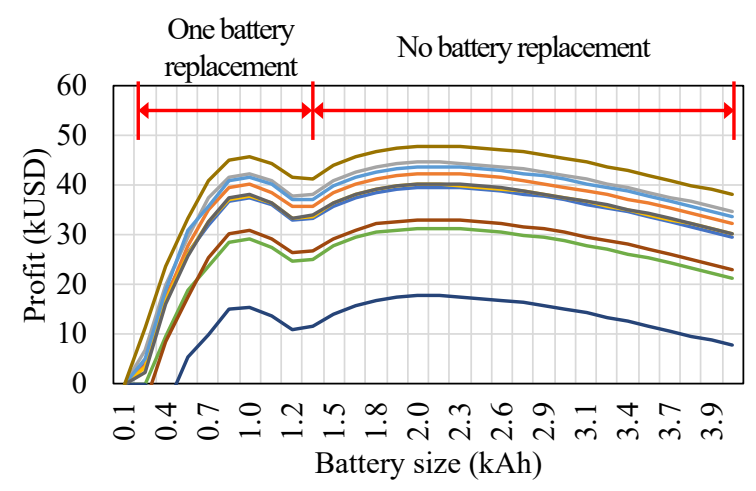

(e) $T=3$ years.

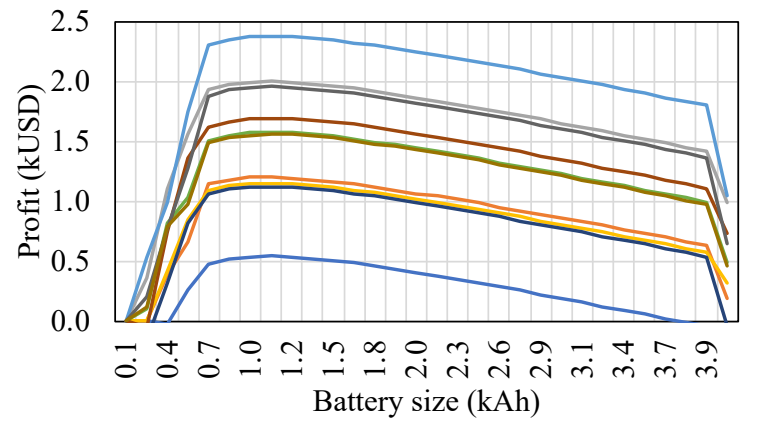

(b) $T=1$ month.

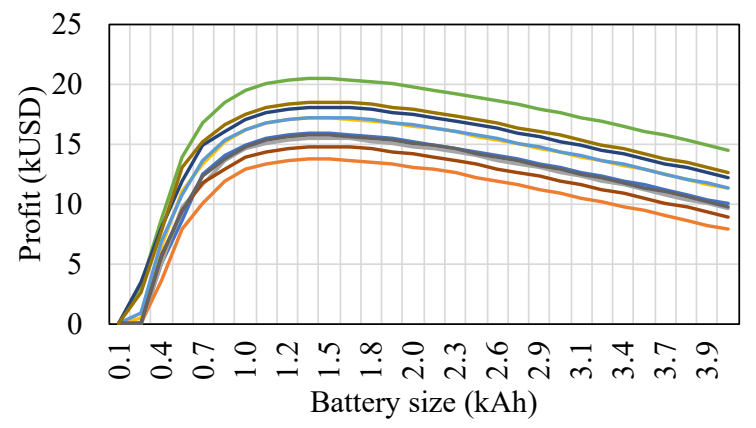

(d) $T=1$ year.

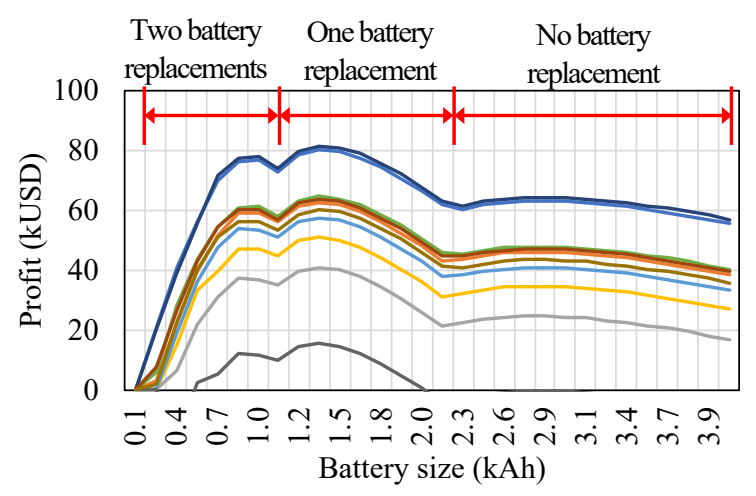

(f) $T=5$ years.

Figure 8. Profit comparison when (a) $T<1$ week, (b) $T<1$ month, (c) $T<6$ months, (d) $T<1$ year, (e) $T<3$ years, and (f) $T<5$ years, respectively.

\subsection{Case Study: Delivery Problem in Real Roads}

In the previous section, the distribution of the locations was synthetically generated in order to allow for an exploration of the different parameters. In this section, we show the application of 
the framework to a real case, consisting of a set of locations taken from a map and for which actual distances and road traffic were taken into account. Only the package set was still generated randomly in the $[0,30 \mathrm{~kg}]$ range of the half-normal distribution. The destinations of the delivery were limited to towns or cities in a province.

\subsubsection{Extraction of Road and Traffic Information}

We used the Piedmont region in Italy as the delivery destinations. There are 10 destinations in Figure 9a including a depot. The depot marked as a red circle was assumed as a UPS delivery center in Torino. There were several route options connecting destinations to each other. Among them, the most recommended one by Google Maps was picked. We extracted the distance of each route and the related driving time. The practical distance of the route and driving time were different by direction. The driving time depended on the time of day. Therefore, we implemented one distance matrix and several driving time matrices by time slots. The average distance of the roads was $44 \mathrm{~km}$, and the average time for driving the roads was $51 \mathrm{~min}$.

Figure $9 \mathrm{~b}$ shows the average time by time slots. Average driving time increased from 07:00 to 09:00 and 17:00 to 19:00 because of rush hour. Therefore, the number of deliveries during rush hour was less than that during other time slots. If we did not consider road traffic, the average driving time was 46 minutes.

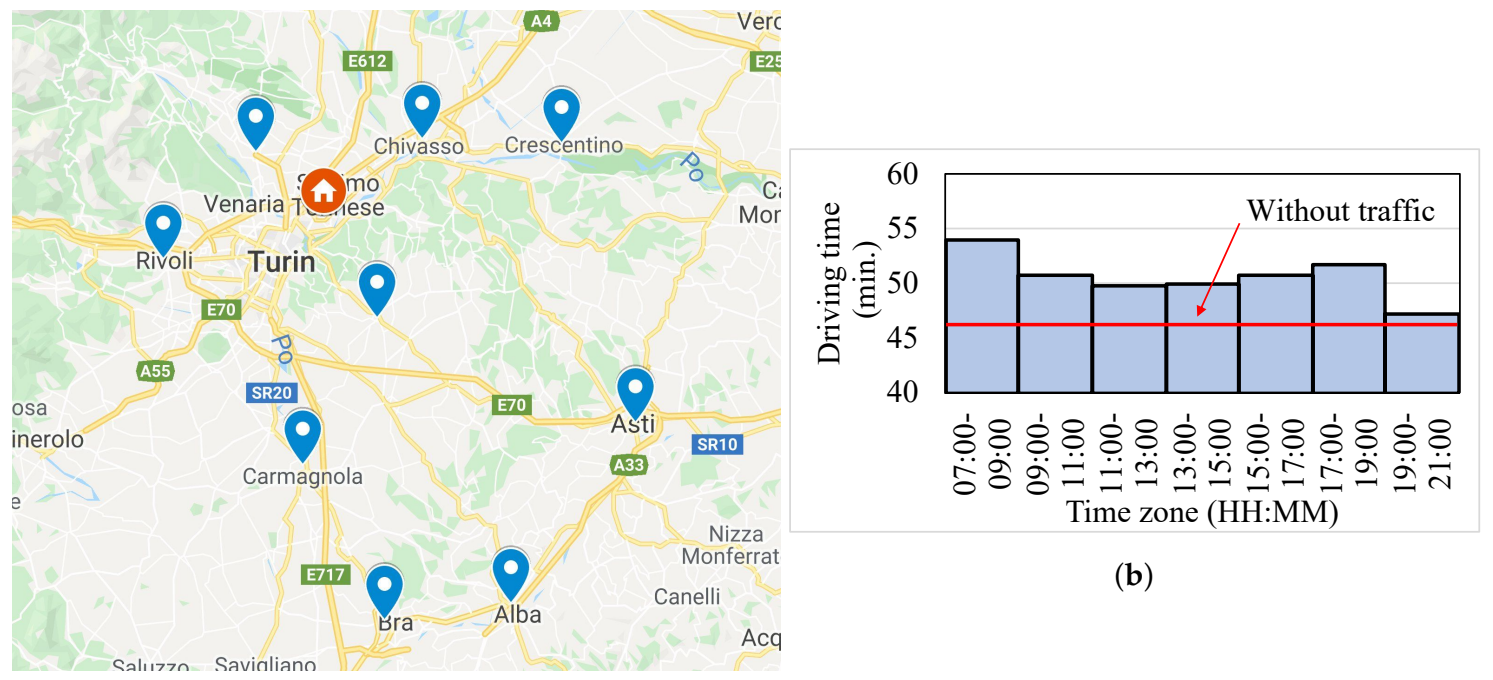

(a)

Figure 9. Delivery problem on real roads: (a) destinations for deliveries and (b) average driving time for moving between destinations.

\subsubsection{Simulation Results}

Figure 10 shows the overall profit of the delivery business by battery size in different working hours. Different colors mean different working hours. In each working hour, there is the optimal battery size maximizing overall profit. The maximum profits without consideration of traffic were $11 \mathrm{kUSD}, 16 \mathrm{kUSD}, 19 \mathrm{kUSD}$, and $21 \mathrm{kUSD}$, respectively. The graphs of the profit shift in the Y direction as we increase the working hours. However, the degree of the shift gradually decreases because the depreciation cost increases more steeply by working hour.

If we considered road traffic, the number of deliveries decreased because of increased driving time. Profit in each working hour also decreased as 8 kUSD, 13 kUSD, 15 kUSD, and 16 kUSD by working hours. 


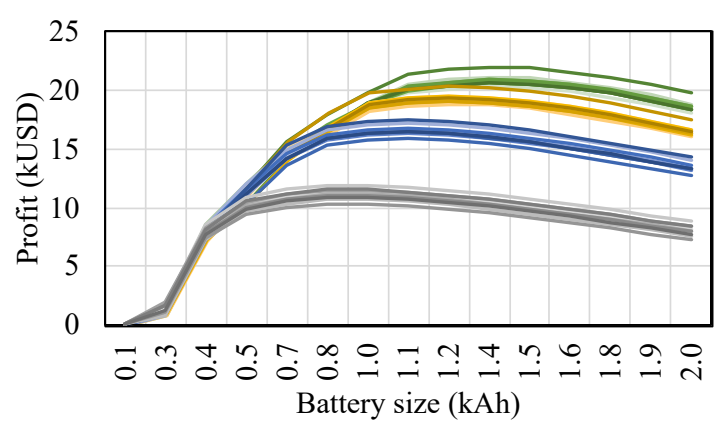

(a) Profit without consideration of road traffic.

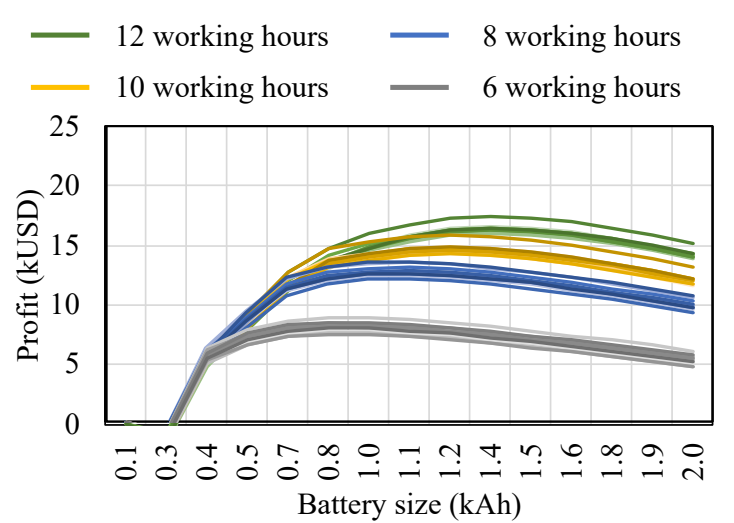

(b) Profit with consideration of road traffic.

Figure 10. Profit comparison by delivery working hour when (a) we do not consider road traffic and

(b) we do consider road traffic.

\section{Conclusions}

A careful battery sizing for electric truck delivery is essential to maximize overall profit considering battery efficiency and degradation. In this work, we proposed a framework for the optimal battery sizing for parcel delivery with an electric truck that included characterizations of (i) vehicle powertrain efficiency, (ii) battery efficiency and degradation, and (iii) delivery requests. We solved the battery sizing problem by maximizing overall profit and analyzed the relationship among delivery payload, battery equivalent cycles, and related battery cost. Our framework could be used for an analysis of the business feasibility under a range of battery sizes and delivery requests. We could estimate the profitability of the delivery business after battery sizing. In addition, our work could be extended as the subject of future work. The profitability could be different under autonomous driving condition. In this case, battery charging time and charging type become concerns instead of working hours.

Author Contributions: D.B. and M.P. outlined the structure and content of this article. N.C. and E.M. proofread the content. D.B. and Y.C. carried out the main experimental works. D.B., Y.C. and M.P. wrote original draft and revised the manuscript. All authors have read and agreed to the published version of the manuscript.

Funding: This work was supported by the National Research Foundation of Korea (NRF) grant funded by the Korea Government (MSIP) (No. NRF- 2018R1A2B3007894).

Conflicts of Interest: The authors declare no conflicts of interest.

\section{References}

1. Green Freight Facts \& Figures. Available online: http://business.edf.org/projects/green-freight-factsfigures (accessed on 1 December 2019).

2. Tesla Press Information. Available online: https://www.tesla.com/presskit\#semi (accessed on 1 December 2019).

3. Chen, Y.; Macii, E.; Poncino, M. A Circuit-equivalent Battery Model Accounting for the Dependency on Load Frequency. In Proceedings of the Conference on Design, Automation \& Test in Europe (DATE), Lausanne, Switzerland, 27-31 March 2017; pp. 1177-1182.

4. Ribau, J.; Silva, C.; Sousa, M. Efficiency, cost and life cycle CO2 optimization of fuel cell hybrid and plug-in hybrid urban buses. Appl. Energy 2014, 129, 320-335. [CrossRef]

5. Song, Z.; Li, J.; Han, X.; Xu, L.; Lu, L.; Ouyang, M.; Hofmann, H. Multi-objective optimization of a semi-active battery/supercapacitor energy storage system for electric vehicles. Appl. Energy 2014, 135, 212-224. [CrossRef]

6. Zhang, L.; Hu, X.; Wang, Z; Sun, F.; Deng, J.; Dorrell, D.G. Multiobjective Optimal Sizing of Hybrid Energy Storage System for Electric Vehicles. IEEE Trans. Veh. Technol. 2018, 67, 1027-1035. [CrossRef] 
7. Sim, K.; Vijayagopal, R.; Kim, N.; Rousseau, A. Optimization of Component Sizing for a Fuel Cell-Powered Truck to Minimize Ownership Cost. Energies 2019, 12, 1125. [CrossRef]

8. Vora, A.P.; Jin, X.; Hoshing, V.; Saha, T.; Shaver, G.; Varigonda, S.; Wasynczuk, O.; Tyner, W.E. Design-space exploration of series plug-in hybrid electric vehicles for medium-duty truck applications in a total cost-of-ownership framework. Appl. Energy 2017, 202, 662-672. [CrossRef]

9. Baek, D.; Chen, Y.; Chang, N.; Macii, E.; Poncino, M. Battery-Aware Electric Truck Delivery Route Planner. ACM/IEEE International Symposium on Low Power Electronics and Design (ISLPED), Lausanne, Switzerland, 29-31 July 2019; pp. 1-6.

10. Baek, D.; Chen, Y.; Bocca, A.; Bottaccioli, L.; Di Cataldo, S.; Gatteschi, V.; Pagliari, D.J.; Patti, E.; Urgese, G.; Chang, N. Battery-Aware Operation Range Estimation for Terrestrial and Aerial Electric Vehicles. IEEE Trans. Veh. Technol. 2019, 68, 5471-5482. [CrossRef]

11. Liu, K.; Zou, C.; Li, K.; Wik, T. Charging Pattern Optimization for Lithium-Ion Batteries With an Electrothermal-Aging Model. IEEE Trans. Ind. Inform. 2018, 14, 5463-5474. [CrossRef]

12. Liu, K.; Hu, X.; Yang, Z.; Xie, Y.; Feng, S. Lithium-ion battery charging management considering economic costs of electrical energy loss and battery degradation. Energy Convers. Manag. 2019, 195, 167-179. [CrossRef]

13. Tesla Model3 Technical Specifications and Performance Figures. Available online: http://www.zeperfs.com/ en/fiche7083-tesla-model-3-75.htm (accessed on 1 December 2019).

14. Tesla Model 3: 2018 Motor Trend Car of The Year Finalist. Available online: https:/ /www.motortrend.com/ news / tesla-model-3-2018-car-of-the-year-finalist/ (accessed on 1 December 2019).

15. Tesla Model 3 \& Chevy Bolt Battery Packs Examined. Available online: https://cleantechnica.com/2018/ 07/08/tesla-model-3-chevy-bolt-battery-packs-examined/ (accessed on 1 December 2019).

16. Mention by Elon Musk in Tweeter. Available online: https://twitter.com/elonmusk/status/ 1117099861273219073 (accessed on 1 December 2019).

17. Prices and Products for Shipping Anywhere in Germany. Deutsche Post DHL Group. 2019. Available online: http:/ / dhlparcel.it/en/privatkunden/preise/preise-national.html (accessed on 1 December 2019).

18. City Performance Scorecards. Available online: https://sfgov.org/scorecards/transportation/congestion (accessed on 1 December 2019).

(C) 2020 by the authors. Licensee MDPI, Basel, Switzerland. This article is an open access article distributed under the terms and conditions of the Creative Commons Attribution (CC BY) license (http:/ / creativecommons.org/licenses/by/4.0/). 